\title{
Functional and structural cortical characteristics after restricted focal motor cortical infarction evaluated at chronic stage - Indications from a preliminary study
}

\author{
Julkunen, Petro
}

2016-08

Julkunen , P , Määttä , S , Säisänen , L, Kallioniemi , E, Könönen , M , Jäkälä , P , Vanninen, R \& Vaalto , S 2016, ' Functional and structural cortical characteristics after restricted focal motor cortical infarction evaluated at chronic stage - Indications from a preliminary study ' , Clinical Neurophysiology , vol. 127 , no. 8 , pp. 2775-2784 . https://doi.org/10.1016/j.clinph.2016

http://hdl.handle.net/10138/224536

https://doi.org/10.1016/j.clinph.2016.05.013

publishedVersion

Downloaded from Helda, University of Helsinki institutional repository.

This is an electronic reprint of the original article.

This reprint may differ from the original in pagination and typographic detail.

Please cite the original version. 


\title{
Functional and structural cortical characteristics after restricted focal motor cortical infarction evaluated at chronic stage - Indications from a preliminary study
}

\author{
Petro Julkunen ${ }^{a, b, *}$, Sara Määttä ${ }^{a, c}$, Laura Säisänen ${ }^{a, c}$, Elisa Kallioniemi ${ }^{\text {a,b }}$, Mervi Könönen ${ }^{\text {a,d }}$, \\ Pekka Jäkälä ${ }^{\mathrm{e}, \mathrm{f}}$, Ritva Vanninen ${ }^{\mathrm{d}, \mathrm{g}}$, Selja Vaalto ${ }^{\mathrm{h}, \mathrm{i}}$ \\ a Department of Clinical Neurophysiology, Kuopio University Hospital, Kuopio, Finland \\ ${ }^{\mathrm{b}}$ Department of Applied Physics, University of Eastern Finland, Kuopio, Finland \\ ${ }^{\mathrm{c}}$ Department of Clinical Neurophysiology, Institute of Clinical Medicine, Faculty of Health Sciences, University of Eastern Finland, Kuopio, Finland \\ ${ }^{\mathrm{d}}$ Department of Clinical Radiology, Kuopio University Hospital, Kuopio, Finland \\ e Department of Neurology, Kuopio University Hospital, Kuopio, Finland \\ ${ }^{\mathrm{f}}$ Department of Neurology, Institute of Clinical Medicine, Faculty of Health Sciences, University of Eastern Finland, Kuopio, Finland \\ ${ }^{\mathrm{g}}$ Department of Clinical Radiology, Institute of Clinical Medicine, Faculty of Health Sciences, University of Eastern Finland, Kuopio, Finland \\ ${ }^{\mathrm{h}}$ Department of Clinical Neurophysiology, Helsinki University Hospital, Helsinki, Finland \\ i Department of Neuroscience and Biomedical Engineering, Aalto University School of Science, Espoo, Finland
}

\section{A R T I C L E I N F O}

\section{Article history:}

Accepted 10 May 2016

Available online 26 May 2016

\section{Keywords:}

Navigated transcranial magnetic

stimulation

Stroke

Infarction

Motor cortex

Motor evoked potential

Diffusion weighted imaging

\section{H I G H L I G H T S}

- TMS and DWI show changes in chronic stroke patients with minor motor symptoms.

- Affected hemisphere is less excitable to produce strong responses.

- At chronic stage, neuronal structure remains disrupted in the affected motor cortex.

\begin{abstract}
A B S T R A C T
Objective: To assess the inter-hemispheric differences in neuronal function and structure of the motor cortex in a small group of chronic stroke patients having suffered a restricted ischemic lesion affecting hand motor representation. GABAergic intracortical inhibition, known to be affected by stroke lesion, was also investigated.

Methods: Eight patients exhibiting little or no motor impairment were studied using transcranial magnetic stimulation (TMS) and diffusion weighted imaging (DWI) $>15$ months from diagnosis. Resting motor threshold (MT) for $50 \mu \mathrm{V}$ and $2 \mathrm{mV}$ motor evoked potentials, and short-interval intracortical inhibition (SICI) were measured from hand muscles. Apparent diffusion coefficients (ADCs) were analyzed from the DWI for the primary motor cortex (M1), the supplementary motor area (SMA) and thalamus for reflecting changes in neuronal organization.

Results: The MTs did not differ between the affected (AH) and unaffected hemisphere (UH) in $50 \mu \mathrm{V}$ responses, while the MTs for $2 \mathrm{mV}$ responses were higher $(p=0.018)$ in AH. SICI was weakened in AH $(p=0.008)$. ADCs were higher in the affected M1 compared to the unaffected M1 $(p=0.018)$ while there were no inter-hemispheric differences in SMA or thalamus.

Conclusions: Inter-hemispheric asymmetry and neuronal organization demonstrated abnormalities in the M1. However, no confident inference can be made whether the observed alterations in neurophysiological and imaging measures have causal role for motor rehabilitation in these patients.

Significance: Neurophysiological changes persist and are detectable using TMS years after stroke even though clinical symptoms have normalized.
\end{abstract}

() 2016 International Federation of Clinical Neurophysiology. Published by Elsevier Ireland Ltd. All rights reserved.

\footnotetext{
* Corresponding author at: Department of Clinical Neurophysiology, Kuopio University Hospital, POB 100, FI-70029 KYS, Kuopio, Finland. Tel.: +358 447174118.

E-mail address: petro.julkunen@kuh.fi (P. Julkunen).
} 


\section{Introduction}

Motor disability is among the most common consequences of ischemic stroke. Following focal ischemic damage, the reorganization of the cortical functions begins (Ward and Cohen, 2004). Some initial improvement after the acute phase occurs due to resolution of the perilesional edema and recovery of other tissue functions surviving the ischemia (Hallett, 2001). However, it is thought that the long-term recovery occurs primarily due to brain plasticity through functional and structural reorganization (Hallett, 2001; Hodics et al., 2006). For example, increased gray matter density and contralesional cortical thickness in the essential cortical motor areas has been correlated with improved arm function in chronic stroke (Gauthier et al., 2008; Sterr et al., 2013).

Lesions in the motor cortex, and the following recovery, cause functional changes in the cortex, in both the affected (AH) and unaffected hemisphere ( $\mathrm{UH})$ as well as in the interhemispheric connections (Chen and Schlaug, 2013; Cunningham et al., 2015; Li et al., 2015; Rossini et al., 2007; Ward and Cohen, 2004). In the acute phase after stroke, the cortical activity increases in the intact non-primary motor and non-motor areas of both hemispheres, whereas later on in well-recovering patients the activity is shifted towards the affected M1 and is reduced in other areas (Calautti et al., 2001a; Calautti et al., 2001b; Feydy et al., 2002; Ward et al., 2003; Ward and Cohen, 2004). The reorganization of the representation area of the paretic hand may occur during the first months after stroke and is usually seen as a mediolateral shift of representation in affected M1. Also an anteroposterior shift of representation towards sensory cortex and premotor areas may be seen (Fridman et al., 2004; Rossini et al., 2007; Traversa et al., 1997) indicating the recruitment of motoneurons from adjacent cortical areas. Interhemispheric inhibition is altered after stroke leading to increased activation in $\mathrm{UH}$ and increased inhibition in $\mathrm{AH}$. Greater imbalance in interhemispheric inhibition and asymmetry in cortical activations has been related to poor recovery (Chen and Schlaug, 2013; Cunningham et al., 2015; Di Lazzaro et al., 1999; Li et al., 2015). Schaechter and Perdue (2008) demonstrated with functional MRI that in stroke patients with good recovery, cortical activity in the $\mathrm{AH}$ motor areas is enhanced depending on the demands of the motor task.

Significant effort has been committed to understanding the neurophysiological mechanisms of functional recovery from brain lesions such as stroke. The region adjacent to lesion demonstrates modified plasticity through remapped sensorimotor functions (Brown et al., 2009; Clarkson et al., 2010; Dijkhuizen et al., 2003). Stroke-related plasticity consists of mechanisms such as early dendritic branching and synaptogenesis, initial increase of cortical excitability via decreased GABA activity enabling use-dependent plasticity, and later on decrease in hyperexcitability (Hagemann et al., 1998; Jones and Schallert, 1992; Neumann-Haefelin et al., 1998; Shimizu et al., 2002; Stroemer et al., 1995; Ward et al., 2003). Stroke is known to increase tonic GABAergic transmission in perilesional cortical area, and a reduction of this inhibition produces early motor recovery after stroke (Clarkson et al., 2010). Counteracting the increased GABAergic inhibition by administering inverse agonist specific for GABA receptors or genetically lowering the number of GABA receptors have been reported to be beneficial for the recovery of motor function after stroke (Clarkson et al., 2010). Hence, the role of the changes in cortical excitability and inhibition in chronic stroke patients with restricted focal lesions and almost complete clinical recovery should be studied more precisely.

Transcranial magnetic stimulation (TMS), a painless noninvasive method, is suitable for investigating the neurophysiological effects of stroke due to its ability to probe corticospinal excitability as well as facilitatory and inhibitory mechanisms of the motor cortex (Rossini et al., 2015). The motor threshold (MT) based on the occurrence of motor evoked potentials (MEPs) induced by TMS is considered to be a common measure of general cortical excitability (Rossini et al., 2015). MT in the affected motor cortex has been shown to increase demonstrating a lowered level of excitation caused by the motor cortex lesion following stroke in the early state (Prashantha et al., 2013). In the long-term recovery, the cortical excitability in $\mathrm{AH}$ has been shown to exhibit a decrease in MT values approaching normal (Takechi et al., 2014; Traversa et al., 2000).

Primary motor cortex disinhibition is a characteristic sign of reorganization in the subacute stage after stroke enabling the recruitment of adjacent motoneurons and facilitating activitydependent plasticity (Liepert et al., 2000). The intracortical GABArelated inhibition, called short-interval intracortical inhibition (SICI), can be studied by paired-pulse TMS using short inter-stimulus intervals (ISIs) (Chen et al., 1998; Kujirai et al., 1993). Intracortical facilitation (ICF) can be assessed with longer ISIs (Kujirai et al., 1993). Previously, SICI and ICF have been studied at different stages of stroke recovery (Cicinelli et al., 2003; Liepert et al., 2000; Malcolm et al., 2015) and a general finding is that SICI is decreased in the $\mathrm{AH}$ and normalization of inhibition is associated with successful recovery (Eliassen et al., 2008; Manganotti et al., 2002; Swayne et al., 2008). Interhemispheric inhibition is often imbalanced in unilateral stroke leading to disinhibition of $\mathrm{UH}$ and increased inhibition of AH through transcallosal fibers (Bütefisch et al., 2003).

In diffusion weighted imaging (DWI) apparent diffusion coefficient (ADC) map reflects the local diffusion of water molecules at each point with a single value (Le Bihan et al., 1986). Thereby in the ADC map, areas with restricted diffusion appear dark while unrestricted diffusion can be seen as bright. The mean ADC values in infarcted neuronal regions change over time; in the acute phase the $\mathrm{ADC}$ decreases, in the subacute phase the ADC returns near to normal values and in the chronic stage the ADC is higher than in the healthy tissue (Shen et al., 2011). Similar change has been observed in thalamus after middle cerebral artery infarcts (Hervé et al., 2005).

The aim of the present study was to understand the chronic phase characteristics of the neurophysiological motor cortical excitability and inhibition in patients with almost complete clinical recovery after restricted focal cortical infarction in the "hand knob" of the primary motor cortex. For this purpose, we studied the excitability, intracortical inhibition and facilitation of the motor cortex using navigated TMS (nTMS) over 15 months after stroke diagnosis in a small group of patients. To gain insight into the relation with parallel cortical anatomical changes, we also assessed ADC to study the local organization of the cortical neurons on the M1, where the lesions were located, and in the supplementary motor area (SMA) and thalamus.

\section{Methods}

\subsection{Patients}

The study was approved by the Research Ethics Committee of Kuopio University Hospital (95/2010). Patients were selected retrospectively from the patient register of Kuopio University Hospital from the years 2005-2009. Criteria for the first selection were the treatment period in the neurology clinic, ICD-10 diagnosis number I63 (stroke) and year of birth 1940 or later. After this, epicrisis from the neurology department and statements of CT- and MR images of selected stroke patients were read. CT and MRIs from patients having first ever unilateral stroke in the immediate vicinity of the anatomical primary hand motor area causing unilateral paresis symptoms in the acute phase were selected for reevaluation by neuroradiologist. This criterion was set to enable the study of long-term plasticity in patients who had clearly intact areas in the close proximity of the lesion site. Selected patients 
were informed about the study and volunteers gave written consent. Altogether eight patients (age: 48-68 years, 6 male) exhibiting little or no motor impairment were recruited for the study 1543 months after having suffered an infarction. The patients were evaluated during a clinical evaluation after diagnosis using modified Rankin Scale (mRS) (van Swieten et al., 1988) and National Institutes of Health Stroke Scoring (NIHSS). None of the patients displayed significant permanent disability ( $m R S \leqslant 1$, NIHHS $\leqslant 1$ ). Due to a retrospective nature of the patient recruitment, no specific tests were used to analyze hand-motor function after the diagnosis.

Patients with epilepsy, metallic implants, a pacemaker, artificial heart valves, a cochlear implant or electronic drug pumps were excluded from the recruitment. Patient demographics are presented in Table 1.

\subsection{Study protocol}

All patients underwent MRI prior to nTMS experiments. Structural T1-weighted 3D MRI and DWI were acquired from all the patients (Philips Achieva 3.0T TX, Philips, The Netherlands) using a SENSE-head-8 coil. The 3D T1-weighted images were obtained with the following parameters: $\mathrm{TR}=8.2 \mathrm{~ms}, \mathrm{TE}=3.7 \mathrm{~ms}$, flip angle $8^{\circ}$, voxel size $=1 \times 1 \times 1 \mathrm{~mm}^{3}$, slice orientation = sagittal, total $=190$ slices. DWI was scanned with an echo planar sequence using the following parameters: $\mathrm{TR}=11953 \mathrm{~ms}$, $\mathrm{TE}=55 \mathrm{~ms}$, flip angle $=90^{\circ}$, voxel size $=2 \times 2 \times 2 \mathrm{~mm}^{3}$, slice orientation $=$ transverse, total $=70$ slices, $b$-values $=0$ and $1000 \mathrm{~mm}^{2} / \mathrm{s}$, number of diffusion gradient directions $=16$.

The nTMS study was conducted using Magstim Bistim stimulator and a figure-eight coil with monophasic waveform (Magstim Company Ltd, Whitland, UK) utilizing the structural T1-weighted MRIs with the neuronavigation (eXimia 3.1, Nexstim Plc, Helsinki, Finland). During stimulation, electromyography (EMG) was recorded from abductor pollicis brevis (APB) muscle with eXimia EMG device (Nexstim Plc, Helsinki, Finland) at $3000 \mathrm{~Hz}$ (filtered to $10-500 \mathrm{~Hz}$ ) using disposable $\mathrm{Ag}-\mathrm{AgCl}$ surface electrodes.

First, the location of the optimal APB muscle representation (hotspot) was mapped, and the optimal coil rotation angle in the tangential plane was selected at the hotspot (Julkunen et al., 2009). This was set as the stimulation target for the following stimulation sequences. At the target, the resting motor threshold (MT50) for the APB was computed with the Rossini-Rothwell method (Rothwell et al., 1999) using acceptance criteria of $50 \mu \mathrm{V}$ for the MEP amplitude. Then, 10 MEPs were induced using 120\% of the MT50 intensity with an inter-trial interval (ITI) of over $5 \mathrm{~s}$ to characterize the mean MEP amplitudes (Julkunen et al., 2012b). Subsequently, a recruitment curve sequence was run with stimulation intensities between $100 \%$ and $150 \%$ of MT50 at $10 \%$

Table 1

Patient demographics.

\begin{tabular}{clllllll}
\hline Id & $\begin{array}{l}\text { Age } \\
\text { (years) }\end{array}$ & Gender & $\begin{array}{l}\text { Affected } \\
\text { hemisphere }\end{array}$ & $\begin{array}{l}\text { Time from } \\
\text { diagnosis } \\
\text { (months) }\end{array}$ & $\begin{array}{l}\text { Carotid } \\
\text { stenosis } \\
\text { (side) }\end{array}$ & mRS & NIHSS \\
\hline 1 & 65 & Male & Right & 31 & Yes (right) & 1 & 0 \\
2 & 66 & Male & Left & 15 & Yes (left) & 0 & 0 \\
3 & 56 & Female & Right & 28 & No & 0 & 0 \\
4 & 65 & Male & Right & 42 & No & 1 & 1 \\
5 & 68 & Female & Left & 42 & No & 0 & 0 \\
6 & 59 & Male & Right & 38 & No & 0 & 0 \\
7 & 48 & Male & Right & 43 & No & 0 & 0 \\
8 & 55 & Male & Right & 37 & No & 1 & 0
\end{tabular}

Abbreviations: mRS, modified Rankin Scale; NIHSS, National Institutes of Health Stroke Scoring. intervals with 10 MEPs induced at each intensity to construct a threshold curve (Julkunen et al., 2011) and the recruitment curve (Devanne et al., 1997; Pitcher et al., 2003). The used ITI was 5$10 \mathrm{~s}$ (Julkunen et al., 2012b). The threshold curve was used to analyze the higher amplitude thresholds with an acceptance criteria of $2 \mathrm{mV}$ (MT2000). Provided that the maximum amplitude of the subject was less $2 \mathrm{mV}$, MT2000 was set at maximum stimulator output, $100 \%$-MSO.

To study the SICI and ICF we used paired-pulse stimulation with $70 \%$ of MT50 as conditioning stimulation intensity and $120 \%$ of MT50 as test pulse intensity (Vaalto et al., 2011). The conditioning pulse was given before the test pulse at three inter-stimulus intervals (ISIs); $2 \mathrm{~ms}$ (SICl2), $3 \mathrm{~ms}$ (SICI3) and $10 \mathrm{~ms}$ (ICF10) (Kujirai et al., 1993). 10 MEPs were recorded with each ISI. The order of the stimulated hemispheres was randomized. Also, the order of the applied paired-pulse sequence (the three different ISIs) was randomized within the investigated hemisphere.

\subsection{Analyses}

MEP amplitudes were analyzed offline using eXimia software by marking the peak-to-peak amplitudes of the MEPs. Peak-topeak muscle activity of $\geqslant 20 \mu \mathrm{V}$ in EMG within $1 \mathrm{~s}$ preceding the MEPs resulted in the rejection of the trial from further analyses. For SICI and ICF effects, MEP amplitudes were normalized to the mean of single-pulse MEPs.

From the DWI data, ADC maps were automatically generated. From the ADC maps, the mean ADC values were calculated with the region of interest (ROI) evaluation of the white matter. Separate ROIs were drawn in M1 for the hand ("hand knob"), SMA and thalamus on both hemispheres (Fig. 1). M1 ROI was placed in the "hand knob" area in the precentral gyrus. SMA ROI was situated on the anterior portion of the paracentral lobule. ROIs were located in the middle slice of the visually estimated slices where the structure, i.e. "hand knob" or SMA, could be detected. Thalamus ROI was located on the slice in which the thalamus reached its maximal size in the axial orientation. Thus, ADC values were evaluated locally from subcortical locations.

\subsection{Statistics}

Interhemispheric differences between $\mathrm{AH}$ and $\mathrm{UH}$ were analyzed using Wilcoxon signed rank test for paired-samples. Indications of potential correlation between time-from-diagnosis (TFD) and interhemispheric differences in measured TMS parameters or ADC were tested using Spearman's rho. Due to a small number of subjects, bootstrap resampling with 10,000 repetitions was used to estimate the $95 \%$ confidence interval $(\mathrm{CI})$ for the significant correlations found. The level for statistical significance was set at $p<0.05$. Analyzes were performed using SPSS (IBM SPSS Statistics, version 22.0, Somers, NY, USA).

\section{Results}

In all patients, the hotspots were located in precentral gyri in both hemispheres and most often in the close vicinity of the "hand knob" lesion in AH (Fig. 2). The MT50s did not differ between the AH and UH hemispheres ( $p>0.999)$, while the MT2000 was higher $(p=0.018)$ in the AH (Figs. 3B, 4A). Five patients exhibited MT2000 of over $100 \%$-MSO in the AH, while one patient exhibited MT2000 of over $100 \%$-MSO in the UH. The threshold curves indicated that the threshold for inducing high amplitude MEPs increased in the AH. MEP amplitudes induced at $120 \%$ rMT were lower $(p=0.012)$ in the $\mathrm{AH}$ (Figs. 3A, 4A).

At the group level, SICI was apparent in the UH, i.e. MEPs were lower in amplitude compared with single-pulse MEPs $(p=0.012$ 

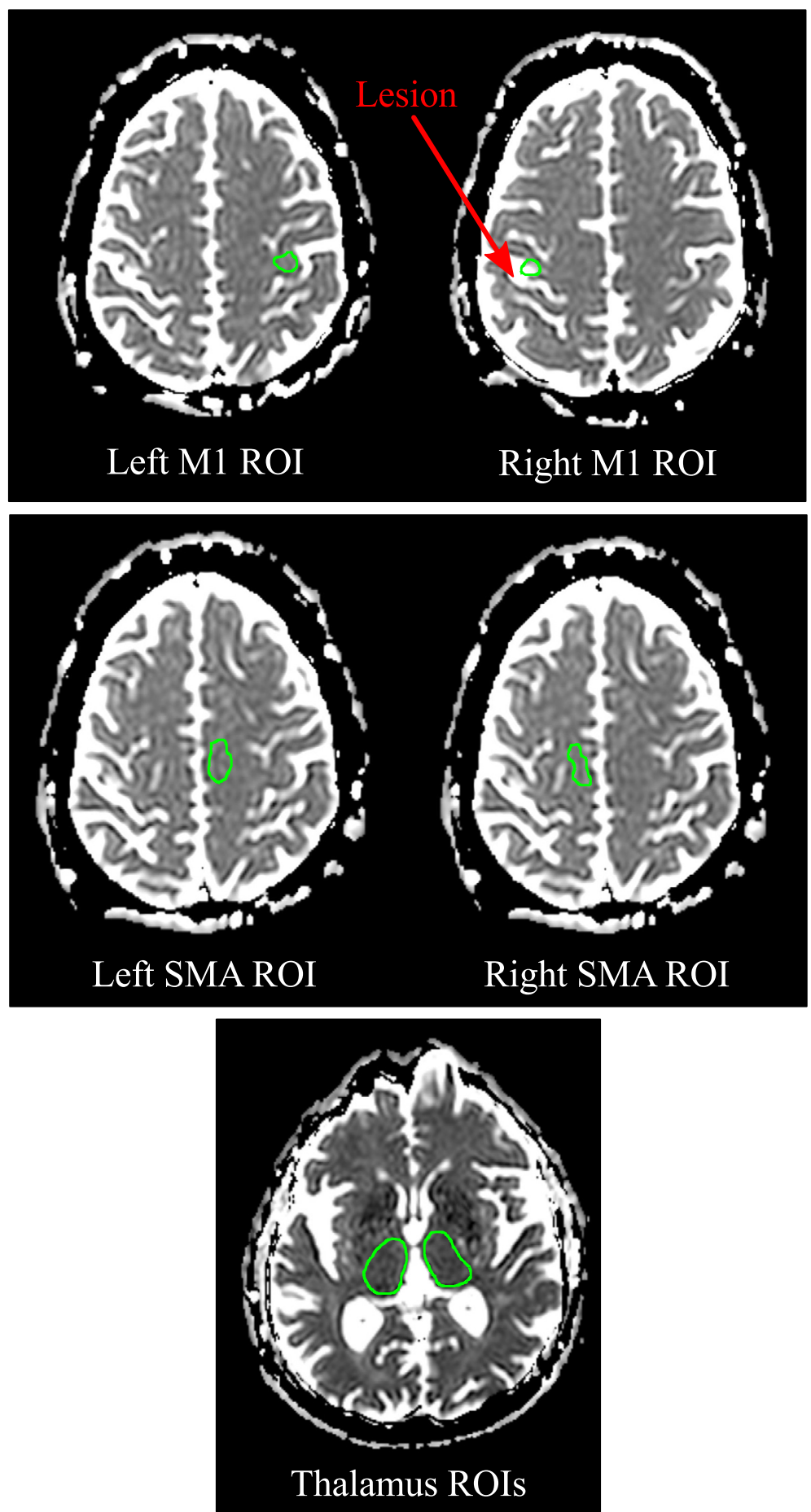

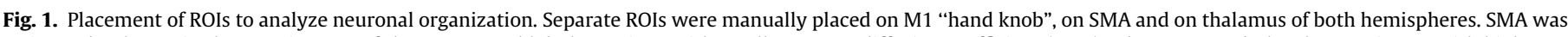

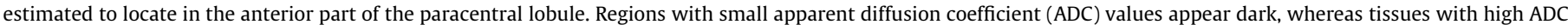
values are bright. Location of the lesion in the right hemisphere "hand knob" has been indicated.

for SICI2 and $p=0.012$ for SICI3) (Fig. 4B). Instead, the SICI was often absent in the AH indicated by a non-significant difference compared with the single-pulse MEPs $(p=0.161$ for SICI2 and $p=0.263$ for SICI3). At the group level, ICF10 effect was nonsignificant in either hemisphere $(p \geqslant 0.327)$. At the individual level, all patients exhibited SICI2 and SICI3, but only 3 exhibited ICF10 in the UH. In the AH, 6 patients exhibited SICI2, 5 patients exhibited SICI3 and 6 patients exhibited ICF10. Individual values for the TMS parameters are shown in Table 2.
DWI was successfully conducted in $7 / 8$ patients. The locations of the ROIs are reported in Table 3. The ADC values in the AH M1 were higher than in the UH M1 $(p=0.018)$, while there were no significant differences between the hemispheres in SMA $(p=0.176)$ or thalamus ( $p=0.498)$ (Fig. 4C). The ADC in the AH side thalamus correlated negatively with the MT2000 ( $r h o=-0.906$, $p=0.005)$ and positively with $\operatorname{MEP}(r h o=0.786, p=0.036)$ in the $\mathrm{AH}$. The correlations were verified by bootstrap resampling with MT2000 (95\% CI for rho $=-1.000$ to $-0.624, p=0.010)$ and MEP 


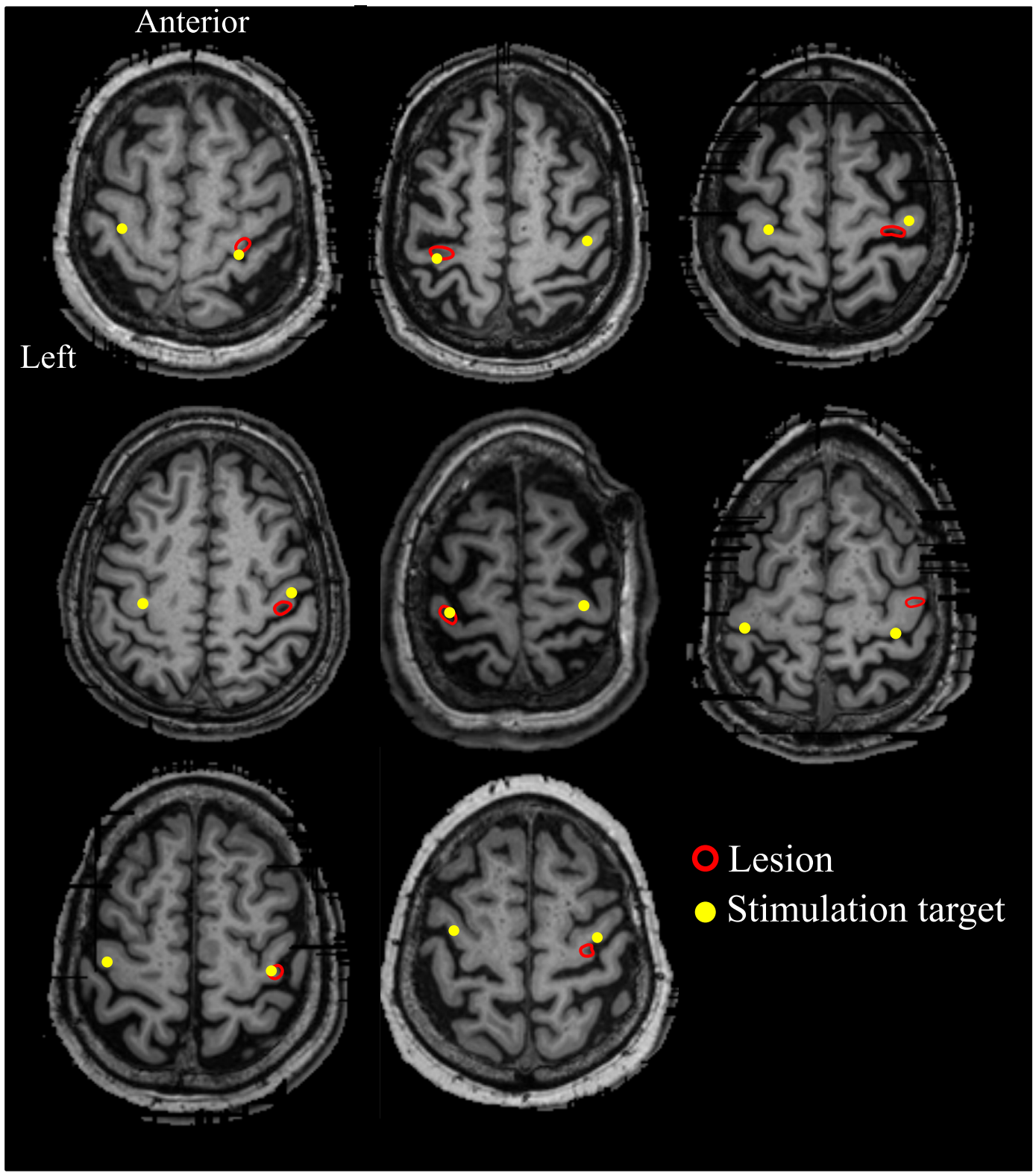

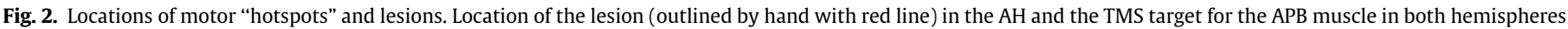

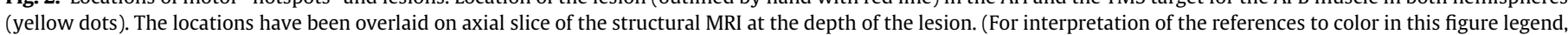
the reader is referred to the web version of this article.)

(95\% CI for $r h o=0.059-1.000, p=0.048)$. No other significant correlations were found between the mean $A D C$ and any of the TMS-measures. Individual ADC values are shown in Table 4.

Correlations were observed between SICI2 and the TFD in both AH ( $r h o=-0.874, p=0.005)$ and $\mathrm{UH}(r h o=-0.762, p=0.028)$. Bootstrap resampling verified our finding in the $\mathrm{AH}(95 \% \mathrm{CI}$ for $r h o=-1.000$ to $-0.333, p=0.005)$ and $\mathrm{UH}(95 \% \mathrm{CI}$ for $r h o=-1.000$ to -0.062 , $p=0.022$ ). Hence, the difference in SICI2 between the $\mathrm{AH}$ and $\mathrm{UH}$ tended to normalize over time, i.e. difference in the SICI2 became smaller ( $r h o=0.881, p=0.004$, Fig. 5 ) again verified by bootstrap resampling $(95 \% \mathrm{CI}$ for $r h o=0.392-1.000, p=0.003$ ). Other TMSparameters or the mean ADC did not exhibit correlation with TFD.

\section{Discussion}

In this study, the functional and structural properties of affected and unaffected motor cortices were studied in chronic stroke patients with unilateral restricted cortical infarctions located in anatomical hand motor representation. We found significant differences between the AH and $\mathrm{UH}$ in cortical excitability, intracortical inhibition as well as in the neuronal organization of M1. The lesions of these patients were small and focused in the immediate vicinity of the anatomical hand motor representation ("hand knob") and little or no symptoms remained at the moment of the study, over 15 months after diagnosis. Therefore, the recovery at the chronic stage, while potentially symptom-free, still seems to remain incomplete, as indicated by the great imbalance in MT2000s between the AH and UH despite overall good interhemispheric symmetry in MT50s. Although no follow-up studies were conducted and the group of patients studied was small, indications of recovery were observed in the inter-individual comparison through SICI2, which showed at the group-level that the intracortical inhibition may be advancing towards normal when more time had passed from diagnosis. However, it must be kept in mind that a low number of subjects were studied and therefore these results are only indications. 
(A)

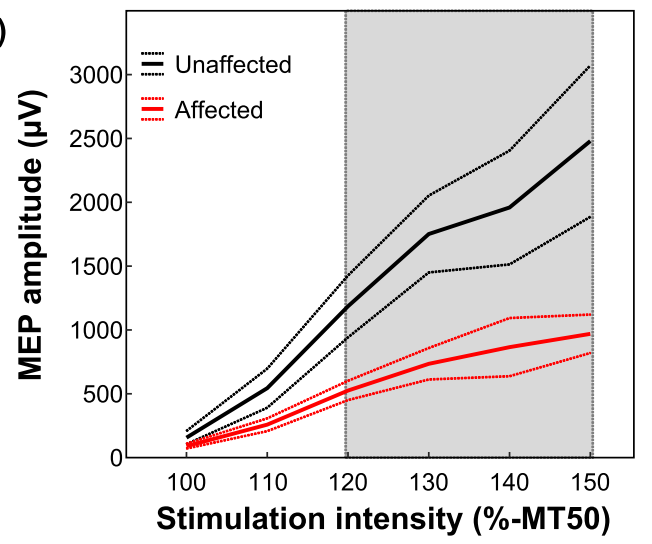

(B)

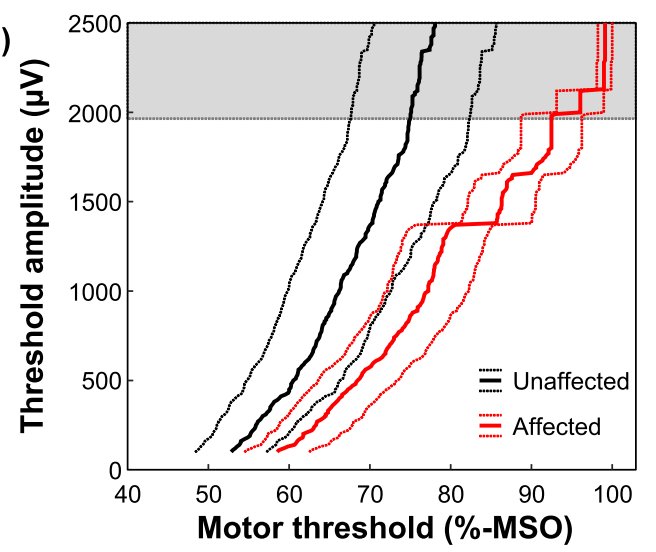

Fig. 3. Recruitment characteristics from TMS. (A) Recruitment curves (mean \pm SEM) for the $\mathrm{AH}$ and $\mathrm{UH}$. The gray areas indicate significant differences between the hemispheres. (B) MEP threshold curves (mean \pm SEM) for the AH and UH. The threshold curve has been constructed by computing the MT using different MEP amplitude threshold criteria after stimulation with several different stimulation intensities (Julkunen et al., 2011). In the AH, not all subjects demonstrated above $2 \mathrm{mV}$ responses meaning that their threshold value MT2000 was $100 \%$-MSO. Thus, a low SEM value at high threshold amplitudes in the $\mathrm{AH}$ reflects reaching maximal motor threshold-value of 100\%-MSO.

The increase in MT is common in the AH in stroke. While we found no significant difference in the conventional resting MT (MT50), we found that the high amplitude MT (MT2000) was significantly higher. This indicates that normal recruitment of cortical motoneurons in suprathreshold (>MT50) stimulation intensities cannot be achieved at the chronic stage, even though the normalization of the low amplitude threshold MT50 had already occurred. Lower MEP amplitudes in the AH agree well with the findings on MTs, and may potentially be explained by different aspects of the tissue damage and plasticity related changes: (1) partial motoneuron loss in cortical motor representation preventing to produce high-amplitude MEPs, (2) disorganization of the cortical structure affecting the proneness to TMS-effects, (3) affected and reorganized subcortical parts of the corticospinal tract, or (4) scattered reorganization of the hand motor representation. Even if motoneuron loss could purely explain increased MT and lower MEP amplitudes in the $\mathrm{AH}$, the $\mathrm{SICI}$ findings potentially suggest the downregulation of the $\mathrm{GABA}_{\mathrm{A}}$ receptor activity, which is known to allow the recruitment of additional motoneurons and reorganization of the motor function (Cicinelli et al., 2003; Hickmott and Merzenich, 2002; Mittmann et al., 1994). Thus, MT and MEPamplitude findings are probably also affected by various plasticity mechanisms. Alternatively, the observed changes in SICI in the $\mathrm{AH}$ could be due to changes in the composition of the corticospinal volley evoked by TMS after stroke.
Measures of motor cortical excitability (e.g. MT) are influenced also by anatomical factors, such as the coil-to-cortex distance affected by distance of scalp from the stimulated cortex (Danner et al., 2012; Julkunen et al., 2012a). In addition, the anatomical neuronal organization and macrostructure may affect the measures of excitability (Janssen et al., 2013, 2014; Kallioniemi et al., 2015b). Hence, stroke-induced changes in any of these anatomical factors may also affect the measured corticospinal excitability and are reflected in the results of the present study.

In the UH, the SICI was observed both with 2 and $3 \mathrm{~ms}$ ISI, but in the $\mathrm{AH}$ it did not appear in the group level indicating a state of disinhibition in the AH. The SICI effect size in the UH was comparable to those measured previously from a healthy population (Kujirai et al., 1993; Säisänen et al., 2011). The observation of the decreased SICI supports the hypothesis that molecular and cellular events of neuronal plasticity are affected perilesionally, and disinhibition is facilitating functional recovery even in the chronic phase (Clarkson et al., 2010). Furthermore, the SICI2 demonstrated inverse correlation with TFD at the group level, providing some indications that the difference between the UH and AH may normalize with time (Fig. 5). Such a relation was not observed with any other parameter. This suggests that cortical disinhibition may be facilitating functional plasticity at least a few years after stroke although the disinhibition could be affected at the corticospinal level after stroke. However, as these correlations were observed with a small group of patients, this should be confirmed with larger patient population. Furthermore, in case of subject 5 (Table 2), SICI2 was 0.00 and SICI3 was 4.56, which might suggest possible interference of short-interval intracortical facilitation (SICF) with SICI, which could appear at ISIs of around $3 \mathrm{~ms}$, but not at an ISI of $2 \mathrm{~ms}$ (Peurala et al., 2008). This was, however, not observed in the other subjects, and requires further study.

We observed that the recruitment curves of the $\mathrm{AH}$ remained at lower amplitude levels than the curves of the UH due to restricted focal lesions (Fig. 3A) similarly to previous stroke studies (Bütefisch et al., 2003; Ward et al., 2006). In the current study, the changes were significant at greater stimulation intensities and higher response amplitudes. This implies that the $\mathrm{AH}$ is unable to recruit as large neuronal population to produce a high amplitude response or powerful movements. Besides motoneuron loss, one explanation could be the nature of reorganization of the motor cortex in a manner that the overlapping representation areas with connecting interneurons and shared descending pathways are not as efficiently activated via focal cortical stimulation based on more scattered and less dense representation areas.

Following stroke, focal reductions in the neuronal tract integrity are observed as the interhemispheric asymmetry of tissue anisotropy. Previous studies have shown that interhemispheric connections between the motor cortices are impaired (Cunningham et al., 2015; Li et al., 2015). A few studies have investigated both TMS and DWI aspects in stroke. Callosal FA has been shown to correlate with motor improvement detected after repetitive-TMS (rTMS) therapy, and higher FA values were associated with a better motor outcome (Demirtas-Tatlidede et al., 2015). In a recent study, Cunningham et al. (2015) reported that patients with greater interhemispheric asymmetry in FA showed greater corticospinal output in the UH. To compare those results with ours, we computed the asymmetry index for ADC and found a similar phenomenon with MEP amplitudes induced at $120 \%$ of MT50 from the recruitment curve (rho $=0.857, p=0.014$ ). This confirms that higher MEP amplitudes evoked by stimulation of $\mathrm{UH}$ were associated with greater asymmetry in ADC values. Greater hemispheric asymmetry in the organization of neuronal fibers may be related to a greater imbalance of interhemispheric inhibition which usually results in decreased inhibition in $\mathrm{UH}$ and increased inhibition in $\mathrm{AH}$. Decreased inhibition and increased activation in the contralesional 
(A)
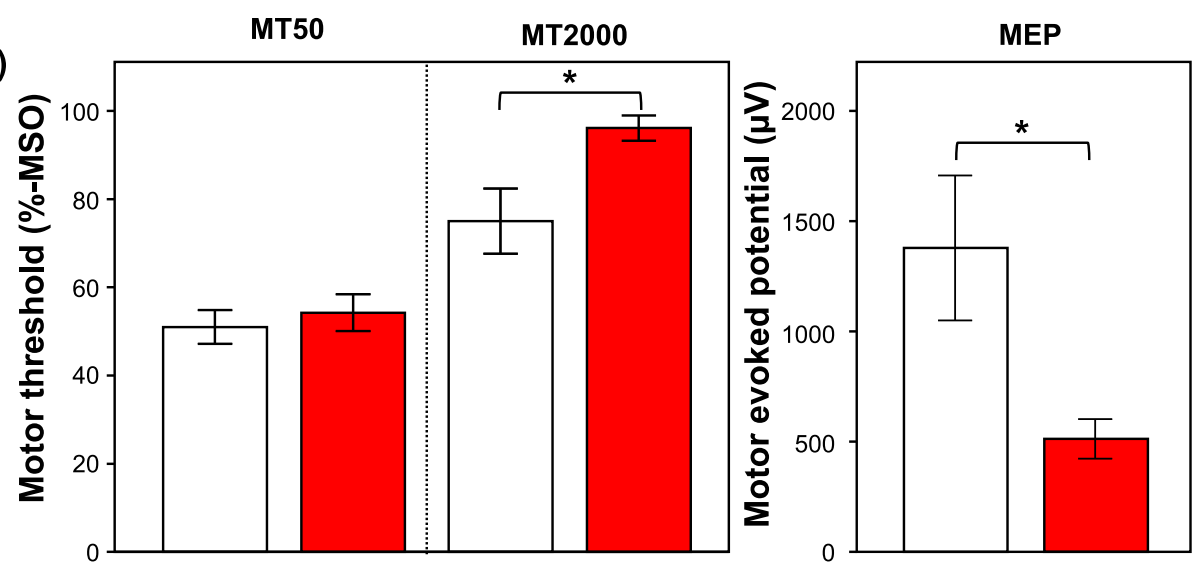

(B)
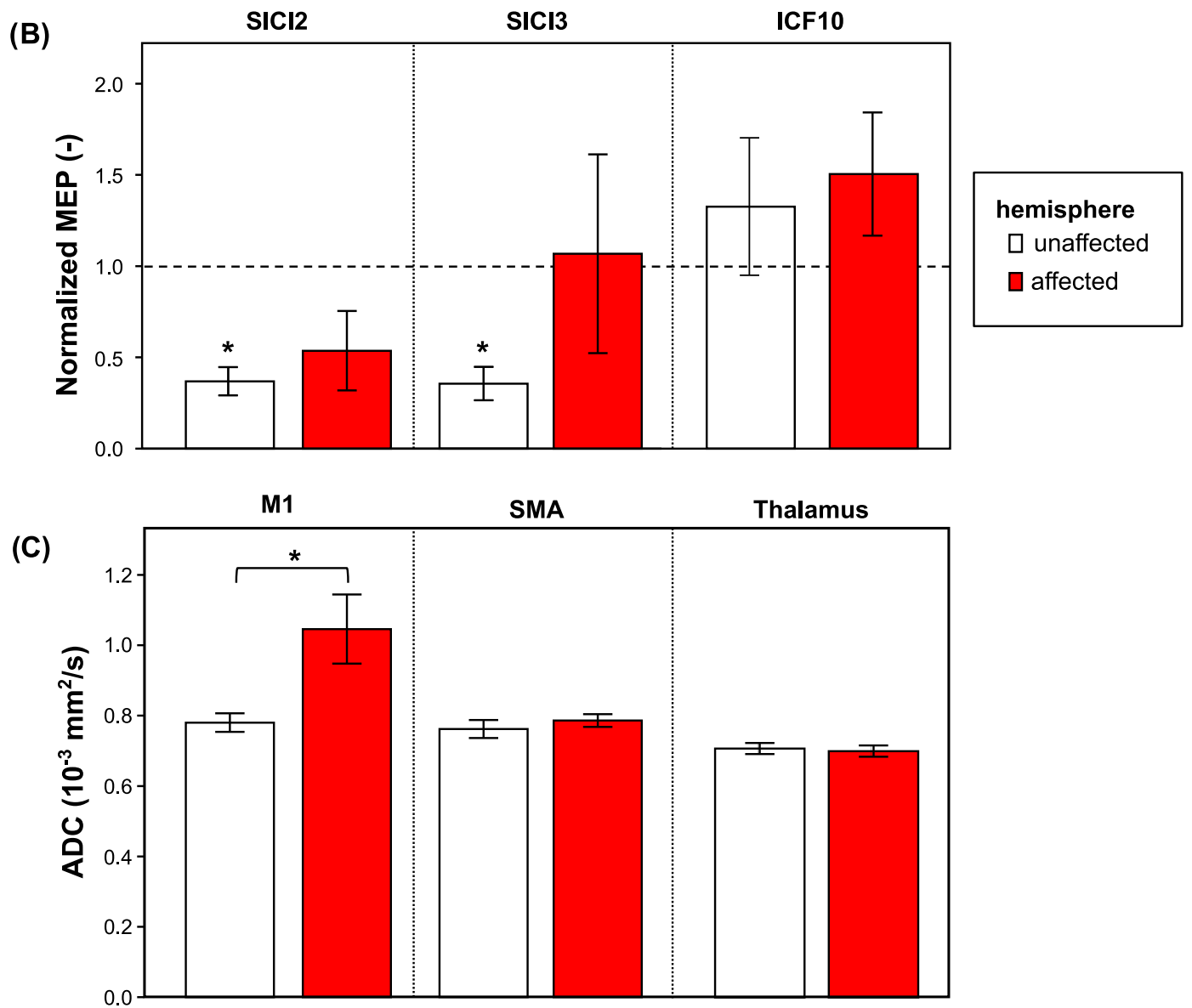

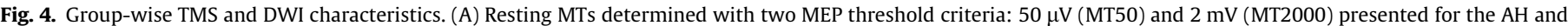

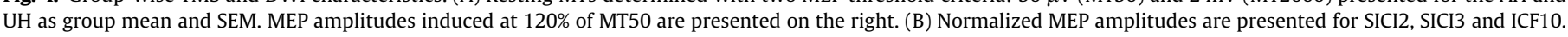

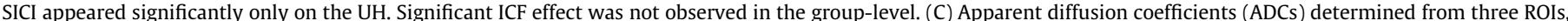

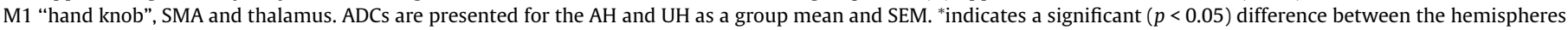
according to the Wilcoxon signed rank test.

motor cortex could explain the finding of more efficient recruitment of motoneurons in $\mathrm{AH}$ than in $\mathrm{UH}$. Interestingly, we found that ADC measured from AH side thalamus correlated positively with the ipsilateral MEP amplitude and negatively with MT2000. It has been shown previously that mean diffusivity in thalamus is greater in healthy controls than in stroke patients in the acute stage while it tends to recover especially on the AH side (Hervé et al., 2005). If we consider that greater MEP amplitude and lower MT2000 are indicative of good recovery, then our results agree. In accordance with previous studies on healthy subjects, we found no other significant correlations between the DWI-derived ADC and the TMS parameters in stroke patients.

Previously, carotid artery stenosis has been shown to affect DWI-weighted MRI and MTs (Avirame et al., 2015; List et al., 2014). In the present study, two patients were observed with hemodynamically relevant carotid artery stenosis (Table 1). Although, we found that stenosis did not clearly affect the MTs or ADC-values (Tables 3 and 4), we made an observation that the two patients diagnosed with relevant stenosis displayed no SICI2 or SICI3. In SICI2, these patients were the only two patients not 
Table 2

Measured TMS characteristics.

\begin{tabular}{|c|c|c|c|c|c|c|c|c|c|c|c|c|}
\hline \multirow[t]{2}{*}{ Id } & \multicolumn{2}{|c|}{ MT50 (\%-MSO) } & \multicolumn{2}{|c|}{$\begin{array}{l}\text { MT2000 (\%- } \\
\text { MSO) }\end{array}$} & \multicolumn{2}{|c|}{$\mathrm{MEP}(\mu \mathrm{V})$} & \multicolumn{2}{|c|}{$\begin{array}{l}\mathrm{SICI} 2 \\
\text { (normalized) }\end{array}$} & \multicolumn{2}{|c|}{$\begin{array}{l}\mathrm{SICI} 3 \\
\text { (normalized) }\end{array}$} & \multicolumn{2}{|c|}{$\begin{array}{l}\text { ICF10 } \\
\text { (normalized) }\end{array}$} \\
\hline & $\mathrm{AH}$ & $\mathrm{UH}$ & $\mathrm{AH}$ & $\mathrm{UH}$ & $\mathrm{AH}$ & $\mathrm{UH}$ & $\mathrm{AH}$ & $\mathrm{UH}$ & $\mathrm{AH}$ & $\mathrm{UH}$ & $\mathrm{AH}$ & $\mathrm{UH}$ \\
\hline 1 & 46 & 45 & 77 & 54 & 610 & 2346 & 1.25 & 0.75 & 1.34 & 0.40 & 1.13 & 0.71 \\
\hline 2 & 73 & 54 & 100 & 100 & 469 & 481 & 1.67 & 0.41 & 1.57 & 0.36 & 1.68 & 0.66 \\
\hline 3 & 51 & 57 & 100 & 90 & 335 & 621 & 0.55 & 0.52 & 0.78 & 0.71 & 2.47 & 3.73 \\
\hline 4 & 62 & 32 & 100 & 49 & 489 & 895 & 0.00 & 0.28 & 0.00 & 0.41 & 1.57 & 0.91 \\
\hline 5 & 45 & 56 & 100 & 72 & 119 & 3207 & 0.00 & 0.11 & 4.56 & 0.11 & 3.13 & 0.75 \\
\hline 6 & 36 & 40 & 100 & 52 & 424 & 1262 & 0.44 & 0.44 & 0.24 & 0.69 & 1.18 & 1.97 \\
\hline 7 & 60 & 63 & 99 & 91 & 681 & 1161 & 0.04 & 0.08 & 0.02 & 0.00 & 0.08 & 1.18 \\
\hline 8 & 61 & 61 & 93 & 92 & 973 & 1060 & 0.34 & 0.36 & 0.03 & 0.17 & 0.80 & 0.70 \\
\hline
\end{tabular}

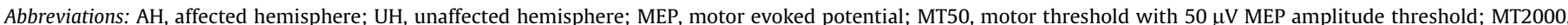

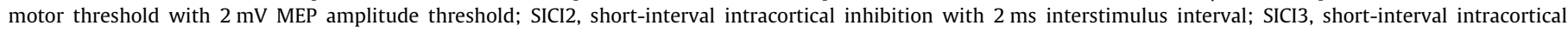
inhibition with $3 \mathrm{~ms}$ interstimulus interval; ICF10, intracortical facilitation with $10 \mathrm{~ms}$ inter-stimulus interval.

Table 3

Individual MNI-coordinates (lateral-medial, anterior-posterior, superior-inferior) in mm for centroids of the ROIs used for the computation of ADC.

\begin{tabular}{|c|c|c|c|c|c|c|}
\hline \multirow[t]{2}{*}{ Id } & \multicolumn{2}{|l|}{ M1 } & \multicolumn{2}{|l|}{ SMA } & \multicolumn{2}{|l|}{ Thalamus } \\
\hline & $\mathrm{AH}$ & $\mathrm{UH}$ & $\mathrm{AH}$ & $\mathrm{UH}$ & $\mathrm{AH}$ & $\mathrm{UH}$ \\
\hline 1 & $30,-20,54$ & $-32,-16,52$ & $12,-10,58$ & $-10,-8,58$ & $16,-14,-2$ & $-16,-14,-2$ \\
\hline 2 & $-34,-18,54$ & $32,-18,42$ & $-10,-6,54$ & $6,-4,54$ & $-16,-14,-4$ & $16,-14,-4$ \\
\hline 3 & $32,-18,50$ & $-34,-22,60$ & $6,-14,56$ & $-14,-12,56$ & $16,-14,-2$ & $-14,-14,-2$ \\
\hline 4 & $32,-16,48$ & $-34,-18,62$ & $10,-8,58$ & $-10,-6,58$ & $16,-14,0$ & $-16,-14,0$ \\
\hline 6 & $34,-16,52$ & $-36,-20,52$ & $8,-6,52$ & $-14,-12,52$ & $14,-12,-2$ & $-14,-14,-2$ \\
\hline 7 & $30,-20,54$ & $-36,-16,54$ & $14,-6,54$ & $-14,-6,54$ & $16,-14,0$ & $-16,-14,0$ \\
\hline 8 & $26,-20,54$ & $-26,-22,54$ & $10,-8,58$ & $-8,-8,58$ & $14,-14,0$ & $-14,-14,0$ \\
\hline
\end{tabular}

Abbreviations: AH, affected hemisphere; UH, unaffected hemisphere; ADC, apparent diffusion coefficient; M1, primary motor cortex; SMA, supplementary motor area.

Table 4

Measured ADC values from DWI.

\begin{tabular}{|c|c|c|c|c|c|c|}
\hline \multirow[t]{2}{*}{ Id } & \multicolumn{2}{|c|}{ ADC on M1 (-) } & \multicolumn{2}{|c|}{ ADC on SMA $(-)$} & \multicolumn{2}{|c|}{$\begin{array}{l}\text { ADC on thalamus } \\
(-)\end{array}$} \\
\hline & $\mathrm{AH}$ & $\mathrm{UH}$ & $\mathrm{AH}$ & $\mathrm{UH}$ & $\mathrm{AH}$ & $\mathrm{UH}$ \\
\hline 1 & 0.886 & 0.851 & 0.835 & 0.849 & 0.719 & 0.696 \\
\hline 2 & 1.403 & 0.851 & 0.792 & 0.862 & 0.703 & 0.692 \\
\hline 3 & 0.818 & 0.764 & 0.802 & 0.727 & 0.663 & 0.704 \\
\hline 4 & 1.406 & 0.737 & 0.729 & 0.702 & 0.697 & 0.716 \\
\hline 6 & 0.877 & 0.694 & 0.746 & 0.699 & 0.701 & 0.720 \\
\hline 7 & 0.846 & 0.712 & 0.745 & 0.727 & 0.711 & 0.691 \\
\hline 8 & 1.085 & 0.853 & 0.852 & 0.769 & 0.712 & 0.740 \\
\hline
\end{tabular}

Abbreviations: AH, affected hemisphere; $\mathrm{UH}$, unaffected hemisphere; ADC, apparent diffusion coefficient; M1, primary motor cortex; SMA, supplementary motor area.

displaying inhibition. While we cannot make a conclusion that stenosis is the reason for the remaining disinhibition, it may be of future research interest.

Previous studies have emphasized the sensitive impact of TMS coil placement and rotation angle to stimulate specific neuronal circuits (Kallioniemi et al., 2015a; Schmidt et al., 2015; Volz et al., 2015). Volz et al. reported that different coil orientations stimulate different neuronal circuits, meaning that the choice of the optimal orientation angle might bias the neuronal circuits investigated. In the present study we utilized nTMS with patientspecific structural MRIs to enable clear anatomical localization of the primary motor cortex and the "hand knob". The optimal coil rotation angle for stimulation was selected based on maximizing the MEP amplitude (Julkunen et al., 2009), and therefore neuronal circuits most directly involved in the execution of muscle movement action were most likely targeted. In most cases the coil rotation angle followed the expected angle across the underlying gyrus, perpendicularly to the closest sulcus. The findings reported may include the limitation that they represent only properties of the neuronal circuits directly involved in the execution of the muscle

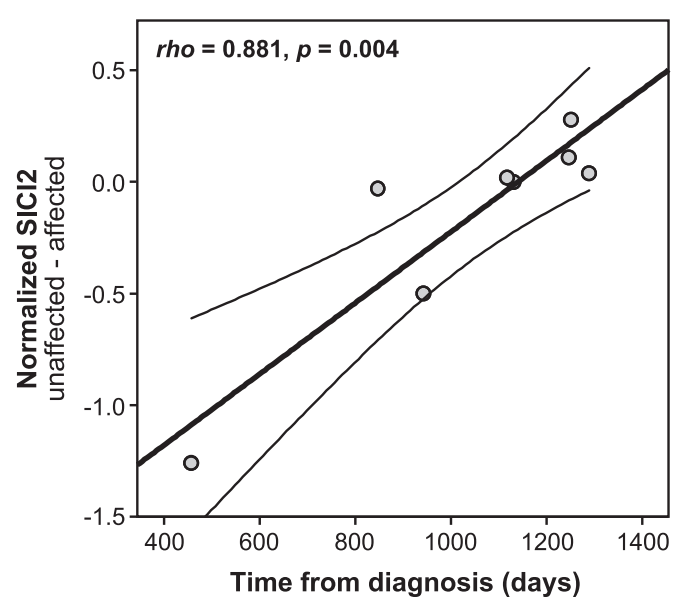

Fig. 5. Group-level recovery of interhemispheric difference in SICI2 over time. Scatter plot between time from diagnosis and interhemispheric difference in SICl effect at $2 \mathrm{~ms}$ ISI. As the time from diagnosis at the time of the study varied between patients, we were able to estimate at the group-level how SICI could recover over time. While the time from diagnosis becomes longer, the SICI effect difference between hemispheres becomes smaller, i.e. the affected side SICI2 tends to normalize. Due to small number of subjects, bootstrap resampling was conducted for the correlation ( $95 \% \mathrm{CI}$ for $r h o=0.392-1.000, p=0.003$ ).

movements, while the other circuits potentially not activated by TMS were not assessed.

Although the characteristics of stroke in terms of lesion location and size as well as induced symptoms were strictly defined in the present study, the demographics of the patients were otherwise heterogeneous. The age of the subjects at the time of measurement varied between 48 and 68 years. Aging may have some effect on the rMT (Säisänen et al., 2008), while contrasting results have been reported (Mills and Nithi, 1997; Wassermann, 2002). The studied population also included female and male subjects. It has been 
reported that there may or may not be differences between genders in rMTs (Mills and Nithi, 1997; Säisänen et al., 2008; Wassermann, 2002), while no significant gender differences have been reported for SICI or ICF (Säisänen et al., 2011; Wassermann, 2002). We also observed large variation in rMT, SICI and ICF in the AH side. Previous reports on inter-hemispheric differences in healthy populations show that no clear inter-hemispheric differences exist in rMT, SICI or ICF (Maeda et al., 2002; Mills and Nithi, 1997; Säisänen et al., 2008, 2011). A recent large-scale study on diffusion tensor imaging on healthy adult subjects reported no gender differences or age-dependency in M1 neuronal arrangement, while mild differences between hemispheres were reported (Jang and Seo, 2015). None of the aforementioned effects alone are such that they can be detected with confidence on the individual level. Therefore, we acknowledge that such factors induce some additional variation to our data and may have prevented some effects caused by stroke recovery from appearing.

The number of patients in the present study was low due to strict criteria set for the patients to specifically study focused motor cortical lesion, and more patients are required to generalize the results. Therefore, the validation of the correlation findings was conducted using the bootstrap resampling, which provides additional confidence in the results. Homogeneity of the patient population studied may have helped to identify some aspects of the motor cortex function and structure in the chronic stage otherwise unidentifiable in a more heterogeneous population, as indicated by the sensitive correlations found between SICI2 and TFD. In addition, the neurological characterization performed using NIHHS and $\mathrm{mRS}$, do not specifically describe the hand function, which limit the analyses of neurophysiological parameters measured with TMS and those experienced by the patients. This was due to the retrospective nature of the patient recruitment, for which reason we had no baseline measures of the hand motor function. Nevertheless, based on the scales used to assess symptom severity, little or no residual motor symptoms were present at the time of study. Due to low number of subjects no confident inference can be made based on this study whether the observed alterations in electrophysiological and imaging measures have causal role for motor rehabilitation in these patients.

The indications provided by the present study motivate for future studies, which should include: (1) subjects which have suffered from focal lesions in the primary motor cortex but have either undergone only partial motor recovery or full motor recovery to enable comparison between the two in progression of motor cortical function and structure, (2) measures able to discriminate between subtle motor impairments (e.g. Action Research Arm Test, ARAT) in order to enable correlating changes in neurophysiological measures with motor function, and (3) study on the specificity of the neurophysiological alterations by comparing different types of impairments (e.g. in speech). Some measurements could also be conducted during a motor task.

\section{Conclusions}

In conclusion, clear changes in the excitability of the AH were observed in this preliminary study with nTMS in a small group of patients expressing little or no remaining symptoms from restricted focal motor cortical infarction. The ability of the $\mathrm{AH}$ motor cortex to respond to activation with higher stimulus intensities was significantly attenuated suggesting that powerful and fine motor movements of the hand were still suffering from the stroke. Functional changes were accompanied by increased diffusion indicating impaired neuronal organization at the site of the lesion. Considering that these changes were observed at chronic stage at least 15 months after diagnosis and that the lesions were focused in M1, the resilient plasticity of the motor cortex to transfer the necessary action functions can be assumed to have occurred. The disinhibition in AH observed even at this stage indicates that excess facilitation of activity-dependent plasticity may still be present, but the normalization of inhibition in a few years' time-window after stroke may potentially be associated to the end of stroke-related functional plasticity. While these findings are indicative of long-term stroke-induced changes in cortical inhibition, proving clinical significance of these findings may require further validation with larger number of patients.

\section{Acknowledgements}

The authors acknowledge the Research Committee of the Kuopio University Hospital Catchment Area for the State Research Funding (projects 5041726, 5041730 and 5041749, Kuopio, Finland).

Conflicts of interest: PJ and SV have received consulting pay from Nexstim Plc., unrelated to this study. The other authors have nothing to disclose.

\section{References}

Avirame K, Lesemann A, List J, Witte AV, Schreiber SJ, Floel A. Cerebral autoregulation and brain networks in occlusive processes of the internal carotid artery. J Cereb Blood Flow Metab 2015;35:240-7.

Brown CE, Aminoltejari K, Erb H, Winship IR, Murphy TH. In vivo voltage-sensitive dye imaging in adult mice reveals that somatosensory maps lost to stroke are replaced over weeks by new structural and functional circuits with prolonged modes of activation within both the peri-infarct zone and distant sites. J Neurosci 2009;29:1719-34.

Bütefisch CM, Netz J, Wessling M, Seitz RJ, Homberg V. Remote changes in cortical excitability after stroke. Brain 2003;126:470-81.

Calautti C, Leroy F, Guincestre JY, Baron JC. Dynamics of motor network overactivation after striatocapsular stroke: a longitudinal PET study using a fixed-performance paradigm. Stroke 2001a;32:2534-42.

Calautti C, Leroy F, Guincestre JY, Marie RM, Baron JC. Sequential activation brain mapping after subcortical stroke: changes in hemispheric balance and recovery. Neuroreport 2001b;12:3883-6.

Chen JL, Schlaug G. Resting state interhemispheric motor connectivity and white matter integrity correlate with motor impairment in chronic stroke. Front Neurol 2013;4:178.

Chen R, Tam A, Butefisch C, Corwell B, Ziemann U, Rothwell JC, Cohen LG. Intracortical inhibition and facilitation in different representations of the human motor cortex. J Neurophysiol 1998;80:2870-81.

Cicinelli P, Pasqualetti P, Zaccagnini M, Traversa R, Oliveri M, Rossini PM. Interhemispheric asymmetries of motor cortex excitability in the postacute stroke stage: a paired-pulse transcranial magnetic stimulation study. Stroke 2003;34:2653-8.

Clarkson AN, Huang BS, Macisaac SE, Mody I, Carmichael ST. Reducing excessive GABA-mediated tonic inhibition promotes functional recovery after stroke. Nature 2010;468:305-9.

Cunningham DA, Machado A, Janini D, Varnerin N, Bonnett C, Yue G, Jones S, Lowe M, Beall E, Sakaie K, Plow EB. Assessment of inter-hemispheric imbalance using imaging and noninvasive brain stimulation in patients with chronic stroke. Arch Phys Med Rehabil 2015;96:S94-103.

Danner N, Könönen M, Säisänen L, Laitinen R, Mervaala E, Julkunen P. Effect of individual anatomy on resting motor threshold-computed electric field as a measure of cortical excitability. J Neurosci Methods 2012;203:298-304.

Demirtas-Tatlidede A, Alonso-Alonso M, Shetty RP, Ronen I, Pascual-Leone A, Fregni F. Long-term effects of contralesional rTMS in severe stroke: safety, cortical excitability, and relationship with transcallosal motor fibers. NeuroRehabilitation 2015;36:51-9.

Devanne H, Lavoie BA, Capaday C. Input-output properties and gain changes in the human corticospinal pathway. Exp Brain Res 1997;114:329-38.

Di Lazzaro V, Oliviero A, Profice P, Saturno E, Pilato F, Tonali P. Motor cortex excitability changes within 8 hours after ischaemic stroke may predict the functional outcome. Eur J Emerg Med 1999;6:119-21.

Dijkhuizen RM, Singhal AB, Mandeville JB, Wu O, Halpern EF, Finklestein SP, Rosen $\mathrm{BR}$, Lo EH. Correlation between brain reorganization, ischemic damage, and neurologic status after transient focal cerebral ischemia in rats: a functional magnetic resonance imaging study. J Neurosci 2003;23:510-7.

Eliassen JC, Boespflug EL, Lamy M, Allendorfer J, Chu WJ, Szaflarski JP. Brainmapping techniques for evaluating poststroke recovery and rehabilitation: a review. Top Stroke Rehabil 2008;15:427-50.

Feydy A, Carlier R, Roby-Brami A, Bussel B, Cazalis F, Pierot L, Burnod Y, Maier MA. Longitudinal study of motor recovery after stroke: recruitment and focusing of brain activation. Stroke 2002;33:1610-7. 
Fridman EA, Hanakawa T, Chung M, Hummel F, Leiguarda RC, Cohen LG. Reorganization of the human ipsilesional premotor cortex after stroke. Brain 2004; $127: 747-58$

Gauthier LV, Taub E, Perkins C, Ortmann M, Mark VW, Uswatte G. Remodeling the brain: plastic structural brain changes produced by different motor therapies after stroke. Stroke 2008;39:1520-5.

Hagemann G, Redecker C, Neumann-Haefelin T, Freund HJ, Witte OW. Increased long-term potentiation in the surround of experimentally induced focal cortical infarction. Ann Neurol 1998;44:255-8.

Hallett M. Plasticity of the human motor cortex and recovery from stroke. Brain Res Brain Res Rev 2001;36:169-74.

Hervé D, Molko N, Pappata S, Buffon F, LeBihan D, Bousser MG, Chabriat H. Longitudinal thalamic diffusion changes after middle cerebral artery infarcts. J Neurol Neurosurg Psychiatry 2005;76:200-5.

Hickmott PW, Merzenich MM. Local circuit properties underlying cortical reorganization. J Neurophysiol 2002;88:1288-301.

Hodics T, Cohen LG, Cramer SC. Functional imaging of intervention effects in stroke motor rehabilitation. Arch Phys Med Rehabil 2006;87:S36-42.

Jang SH, Seo JP. Aging of corticospinal tract fibers according to the cerebral origin in the human brain: a diffusion tensor imaging study. Neurosci Lett 2015;585:77-81.

Janssen AM, Rampersad SM, Lucka F, Lanfer B, Lew S, Aydin U, Wolters CH, Stegeman DF, Oostendorp TF. The influence of sulcus width on simulated electric fields induced by transcranial magnetic stimulation. Phys Med Biol 2013;58:4881-96.

Janssen AM, Oostendorp TF, Stegeman DF. The effect of local anatomy on the electric field induced by TMS: evaluation at 14 different target sites. Med Biol Eng Comput 2014;52:873-83.

Jones TA, Schallert T. Overgrowth and pruning of dendrites in adult rats recovering from neocortical damage. Brain Res 1992;581:156-60.

Julkunen P, Säisänen L, Danner N, Niskanen E, Hukkanen T, Mervaala E, Könönen M. Comparison of navigated and non-navigated transcranial magnetic stimulation for motor cortex mapping, motor threshold and motor evoked potentials. Neuroimage 2009;44:790-5.

Julkunen P, Ruohonen J, Sääskilahti S, Säisänen L, Karhu J. Threshold curves for transcranial magnetic stimulation to improve reliability of motor pathway status assessment. Clin Neurophysiol 2011;122:975-83.

Julkunen P, Säisänen L, Danner N, Awiszus F, Könönen M. Within-subject effect of coil-to-cortex distance on cortical electric field threshold and motor evoked potentials in transcranial magnetic stimulation. J Neurosci Methods 2012a;206:158-64.

Julkunen P, Säisänen L, Hukkanen T, Danner N, Könönen M. Does second-scale intertrial interval affect motor evoked potentials induced by single-pulse transcranial magnetic stimulation? Brain Stimul 2012b;5:526-32.

Kallioniemi E, Könönen M, Julkunen P. Repeatability of functional anisotropy in navigated transcranial magnetic stimulation - coil-orientation versus response. Neuroreport 2015a;26:515-21.

Kallioniemi E, Könönen M, Säisänen L, Gröhn H, Julkunen P. Functional neuronal anisotropy assessed with neuronavigated transcranial magnetic stimulation. J Neurosci Methods 2015b;256:82-90.

Kujirai T, Caramia MD, Rothwell JC, Day BL, Thompson PD, Ferbert A, Wroe S, Asselman P, Marsden CD. Corticocortical inhibition in human motor cortex. J Physiol 1993;471:501-19.

Le Bihan D, Breton E, Lallemand D, Grenier P, Cabanis E, Laval-Jeantet M. MR imaging of intravoxel incoherent motions: application to diffusion and perfusion in neurologic disorders. Radiology 1986;161:401-7.

Li Y, Wu P, Liang F, Huang W. The microstructural status of the corpus callosum is associated with the degree of motor function and neurological deficit in stroke patients. PLoS One 2015;10:e0122615.

Liepert J, Hamzei F, Weiller C. Motor cortex disinhibition of the unaffected hemisphere after acute stroke. Muscle Nerve 2000;23:1761-3.

List J, Hertel-Zens S, Kubke JC, Lesemann A, Schreiber SJ, Floel A. Cortical reorganization due to impaired cerebral autoregulation in individuals with occlusive processes of the internal carotid artery. Brain Stimul 2014;7:381-7.

Maeda F, Gangitano M, Thall M, Pascual-Leone A. Inter- and intra-individual variability of paired-pulse curves with transcranial magnetic stimulation (TMS). Clin Neurophysiol 2002;113:376-82.

Malcolm MP, Vaughn HN, Greene D. Inhibitory and excitatory motor cortex dysfunction persists in the chronic poststroke recovery phase. J Clin Neurophysiol 2015;32:251-6.

Manganotti P, Patuzzo S, Cortese F, Palermo A, Smania N, Fiaschi A. Motor disinhibition in affected and unaffected hemisphere in the early period of recovery after stroke. Clin Neurophysiol 2002;113:936-43.

Mills KR, Nithi KA. Corticomotor threshold to magnetic stimulation: normal values and repeatability. Muscle Nerve 1997;20:570-6.

Mittmann T, Luhmann HJ, Schmidt-Kastner R, Eysel UT, Weigel H, Heinemann U. Lesion-induced transient suppression of inhibitory function in rat neocortex in vitro. Neuroscience 1994;60:891-906.

Neumann-Haefelin T, Staiger JF, Redecker C, Zilles K, Fritschy JM, Mohler H, Witte OW. Immunohistochemical evidence for dysregulation of the GABAergic system ipsilateral to photochemically induced cortical infarcts in rats. Neuroscience 1998:87:871-9.

Peurala SH, Muller-Dahlhaus JF, Arai N, Ziemann U. Interference of short-interval intracortical inhibition (SICI) and short-interval intracortical facilitation (SICF) Clin Neurophysiol 2008;119:2291-7.

Pitcher JB, Ogston KM, Miles TS. Age and sex differences in human motor cortex input-output characteristics. J Physiol 2003;546:605-13.

Prashantha DK, Sriranjini SJ, Sathyaprabha TN, Nagaraja D, Pal PK. Evaluation of the motor cortical excitability changes after ischemic stroke. Ann Indian Acad Neurol 2013;16:394-7.

Rossini PM, Altamura C, Ferreri F, Melgari JM, Tecchio F, Tombini M, Pasqualetti P Vernieri F. Neuroimaging experimental studies on brain plasticity in recovery from stroke. Eura Medicophys 2007;43:241-54.

Rossini PM, Burke D, Chen R, Cohen LG, Daskalakis Z, Di Iorio R, Di Lazzaro V, Ferreri F, Fitzgerald PB, George MS, Hallett M, Lefaucheur JP, Langguth B, Matsumoto H, Miniussi C, Nitsche MA, Pascual-Leone A, Paulus W, Rossi S, Rothwell JC, Siebner HR, Ugawa Y, Walsh V, Ziemann U. Non-invasive electrical and magnetic stimulation of the brain, spinal cord, roots and peripheral nerves: Basic principles and procedures for routine clinical and research application. An updated report from an I.F.C.N. Committee. Clin Neurophysiol 2015;126:1071-107.

Rothwell JC, Hallett M, Berardelli A, Eisen A, Rossini P, Paulus W. Magnetic stimulation: motor evoked potentials. The International Federation of Clinical Neurophysiology. Electroencephalogr Clin Neurophysiol Suppl 1999;52:97-103.

Säisänen L, Julkunen P, Niskanen E, Danner N, Hukkanen T, Lohioja T, Nurkkala J, Mervaala E, Karhu J, Könönen M. Motor potentials evoked by navigated transcranial magnetic stimulation in healthy subjects. J Clin Neurophysiol 2008;25:367-72.

Säisänen L, Julkunen P, Niskanen E, Hukkanen T, Mervaala E, Karhu J, Könönen M. Short- and intermediate-interval cortical inhibition and facilitation assessed by navigated transcranial magnetic stimulation. J Neurosci Methods 2011;195:241-8.

Schaechter JD, Perdue KL. Enhanced cortical activation in the contralesional hemisphere of chronic stroke patients in response to motor skill challenge. Cereb Cortex 2008;18:638-47.

Schmidt S, Bathe-Peters R, Fleischmann R, Ronnefarth M, Scholz M, Brandt SA Nonphysiological factors in navigated TMS studies; confounding covariates and valid intracortical estimates. Hum Brain Mapp 2015;36:40-9.

Shen JM, Xia XW, Kang WG, Yuan JJ, Sheng L. The use of MRI apparent diffusion coefficient $(A D C)$ in monitoring the development of brain infarction. BMC Med Imaging 2011;11:2

Shimizu T, Hosaki A, Hino T, Sato M, Komori T, Hirai S, Rossini PM. Motor cortical disinhibition in the unaffected hemisphere after unilateral cortical stroke. Brain 2002;125:1896-907.

Sterr A, Dean PJ, Vieira G, Conforto AB, Shen S, Sato JR. Cortical thickness changes in the non-lesioned hemisphere associated with non-paretic arm immobilization in modified CI therapy. Neuroimage Clin 2013;2:797-803.

Stroemer RP, Kent TA, Hulsebosch CE. Neocortical neural sprouting, synaptogenesis and behavioral recovery after neocortical infarction in rats. Stroke $1995 ; 26: 2135-44$.

Swayne OB, Rothwell JC, Ward NS, Greenwood RJ. Stages of motor output reorganization after hemispheric stroke suggested by longitudinal studies of cortical physiology. Cereb Cortex 2008;18:1909-22.

Takechi U, Matsunaga K, Nakanishi R, Yamanaga H, Murayama N, Mafune K, Tsuji S. Longitudinal changes of motor cortical excitability and transcallosal inhibition after subcortical stroke. Clin Neurophysiol 2014;125:2055-69.

Traversa R, Cicinelli P, Bassi A, Rossini PM, Bernardi G. Mapping of motor cortical reorganization after stroke. A brain stimulation study with focal magnetic pulses. Stroke 1997;28:110-7.

Traversa R, Cicinelli P, Oliveri M, Giuseppina Palmieri M, Filippi MM, Pasqualetti P, Rossini PM. Neurophysiological follow-up of motor cortical output in stroke patients. Clin Neurophysiol 2000;111:1695-703.

Vaalto S, Säisänen L, Könönen M, Julkunen P, Hukkanen T, Määttä S, Karhu J Corticospinal output and cortical excitation-inhibition balance in distal hand muscle representations in nonprimary motor area. Hum Brain Mapp 2011;32:1692-703.

van Swieten JC, Koudstaal PJ, Visser MC, Schouten HJ, van Gijn J. Interobserver agreement for the assessment of handicap in stroke patients. Stroke 1988;19:604-7.

Volz LJ, Hamada M, Rothwell JC, Grefkes C. What makes the muscle twitch: motor system connectivity and TMS-induced activity. Cereb Cortex 2015;25:2346-53.

Ward NS, Cohen LG. Mechanisms underlying recovery of motor function after stroke. Arch Neurol 2004;61:1844-8.

Ward NS, Brown MM, Thompson AJ, Frackowiak RS. Neural correlates of motor recovery after stroke: a longitudinal fMRI study. Brain 2003;126:2476-96.

Ward NS, Newton JM, Swayne OB, Lee L, Thompson AJ, Greenwood RJ, Rothwell JC Frackowiak RS. Motor system activation after subcortical stroke depends on corticospinal system integrity. Brain 2006;129:809-19.

Wassermann EM. Variation in the response to transcranial magnetic brain stimulation in the general population. Clin Neurophysiol 2002;113:1165-71. 